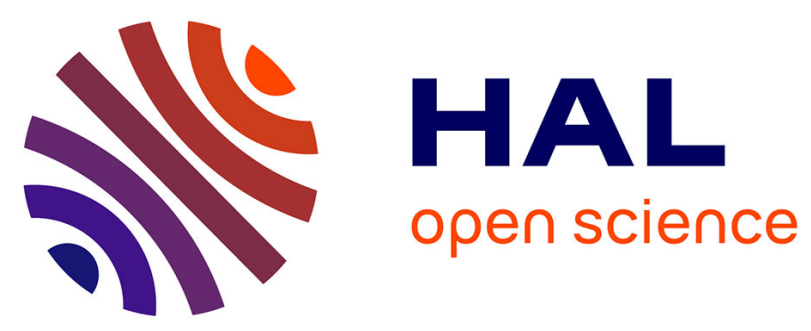

\title{
Parametric Inversion of Brillouin Spectra to Enhance the Accuracy of Distributed Strain Measurement
}

\author{
Edouard Buchoud, Jerome I. Mars, Valeriu Vrabie, Guy d'Urso, Sylvain
} Blairon, Jean-Marie Henault, Alexandre Girard

\section{To cite this version:}

Edouard Buchoud, Jerome I. Mars, Valeriu Vrabie, Guy d'Urso, Sylvain Blairon, et al.. Parametric Inversion of Brillouin Spectra to Enhance the Accuracy of Distributed Strain Measurement. EAGE 2014 - 76th European Association of Geoscientists and Engineers Conference and Exhibition, Jun 2014, Amsterdam, Netherlands. pp.Th G102 09. hal-01018812

\section{HAL Id: hal-01018812 https://hal.science/hal-01018812}

Submitted on 5 Jul 2014

HAL is a multi-disciplinary open access archive for the deposit and dissemination of scientific research documents, whether they are published or not. The documents may come from teaching and research institutions in France or abroad, or from public or private research centers.
L'archive ouverte pluridisciplinaire HAL, est destinée au dépôt et à la diffusion de documents scientifiques de niveau recherche, publiés ou non, émanant des établissements d'enseignement et de recherche français ou étrangers, des laboratoires publics ou privés. 


\title{
Th G102 09
}

\section{Parametric Inversion of Brillouin Spectra to Enhance the Accuracy of Distributed Strain Measurement}

\author{
E. Buchoud* (EDF), J. Mars (GIPSA-LAB), V. Vrabie (CRESTIC), G. D'urso \\ (EDF), S. Blairon (EDF), J.M. Henault (EDF) \& A. Girard (EDF)
}

\section{SUMMARY}

To ensure stability and durability of engineering structure in natural soil, optical fiber sensors have gained interest over last decade. In addition to conventional geophysical sensors, Brillouin spectra based sensor enables to perform distributed strain measurement. Its algorithm performs a strain measurement with a $40 \mathrm{~cm}$ spatial sampling over several kilometers. The monitoring of engineering installations needs a centimeter spatial sampling and a better strain accuracy. Previous works highlighted that the industrialized algorithm has great limitation for the exploitation of the local information contained into Brillouin spectra. Indeed, based on its asymmetry and broadening, it is possible to estimate local Brillouin frequencies with a better strain accuracy. We propose here to apply a parametric inverse method using L-curve criterion to estimate the strain with a $5 \mathrm{~cm}$ spatial sampling. To validate this method, a one-to-one scale experiment has been implemented by optical fiber cable at several depths. Comparing the distributed strain provided by the Brillouin based sensor and our algorithm with a reference strain sensor, the proposed algorithm successfully fulfills the combination of a $5 \mathrm{~cm}$ spatial sampling over kilometers and a high strain accuracy. 


\section{Introduction}

Geotechnical structures as canals or dykes suffer from long term effects as internal erosion could provoke water leakage or embedded cavities. To ensure their durability and stability, the French electric utility company EDF (Electricité de France) chose an innovative structural health monitoring policy to perform strain measurements. It consists in using Distributed Fiber Optic Sensors (DOFS) in addition to conventional sensors.

DOFS, which have gained interests even if many challenges are still opened as referenced by Lanticq et al. (2009), are composed by an opto-electronic device linked to an optical fibre cable, the sensing element. A frequency spanned light impulsion is launched into the medium and the backscatter light recorded by the device allows measuring strain and/or temperature profiles along the optical fiber. Three scattering phenomena are observed: the Raman (temperature sensitive), Brillouin and Rayleigh effects (both strain and temperature sensitive).The Brillouin Optical Time Domain Analyzer (BOTDA) records several Brillouin spectra matrices, centered in the Brillouin frequencies and depending on distance along the optical fiber. As these frequencies are linked directly to the strain imposed to the optical fiber, industrial BOTDA devices estimate a strain profile typically with a spatial sampling of $40 \mathrm{~cm}$ over $25 \mathrm{~km}$ (Bao et al., 1995). However, previous work (Buchoud et al., 2012) highlights the fact that firstly, the spectra could be distorted by non-uniform strain distribution along the fiber and, secondly, that the spatial resolution is limited.

We propose in this article an alternative algorithm to perform and reach a centimeter spatial resolution strain estimation from the measured Brillouin matrices. Thus, the detection and localization of strain gradients into structures will be upgraded. To fulfil such goal, a model proposed in Buchoud et al., 2013 explains the link between the hidden information inside Brillouin spectra and the local Brillouin frequencies. A parametric inverse methodology is proposed to estimate these local frequencies.

A laboratory test with an optical fiber embedded into a one-to-one scale soil structure exposed by Blairon et al. (2011) enabled to validate our algorithm. First, a strain profile with $3 \mathrm{~cm}$ spatial resolution provided by a Rayleigh device is considered here as the reference profile. Secondly, a classical BOTDA device records the Brillouin matrix with a $40 \mathrm{~cm}$ spatial sampling. We show that from those spectra, the proposed algorithm enables to take advantage of the hidden information contained into the distorted spectra to improve the accuracy of the strain measurement and the spatial sampling form $40 \mathrm{~cm}$ to $5 \mathrm{~cm}$.

\section{The BOTDA principle and model}

The BOTDA is based on the stimulated Brillouin scattering (SBS): a light pulse is launched into an optical fibre. The travel time is related to localization $\mathrm{z}$ along the fiber, while the duration of the impulsion is related to the area interrogated into the fiber, called the integration base $w$. For example, for $1 \mathrm{~ns}$ light pulse duration, $1 \mathrm{~m}$ of the sensing medium is interrogated. The light frequency $v$ is spanned and a Brillouin spectrum $G(v)$ is acquired at $z$. The measurement operation is repeated at different localizations $\mathrm{z}$ spaced by a spatial sampling $\Delta z$, allowing to obtain a matrix of Brillouin spectra $G(v, z)$. For each $z$, an industrial BOTDA device automatically finds the global Brillouin frequency $v_{B}(z)$, determined by the best fitting the Brillouin spectrum, in terms of L2 norm, with a pseudo-Voigt function. From a state (without strain for instance) to another one (with strain), the relative Brillouin shift $\Delta v_{B}(z)$ between these two states is linearly related to the strain $\boldsymbol{\varepsilon}(\mathbf{z})$ and temperature $\Delta \mathrm{T}(\mathrm{z})$ conditions along the fiber optic (Bao et al., 1995) as:

$$
\Delta v_{\mathrm{B}}(\mathrm{z})=\mathrm{C}_{\varepsilon} \cdot \varepsilon(\mathrm{z})+\mathrm{C}_{\mathrm{T}} \cdot \Delta \mathrm{T}(\mathrm{z})
$$

where $\mathrm{C}_{\mathrm{T}}$ and $\mathrm{C}_{\varepsilon}$ are about $1 \mathrm{MHz} /{ }^{\circ} \mathrm{C}$ and $0.05 \mathrm{MHz} / \mu \varepsilon$ for a standard single-mode optical fiber with an operating wavelength of $1550 \mathrm{~nm}$. In our study, since the temperature $\mathrm{T}(\mathrm{z})$ is constant for all measurements, the strain $\varepsilon(\mathrm{z})$ can be directly deduced from (1).

However, as the BOTDA integrates over 1 meter at each $z$, it is possible to mix non-uniform local strain within $w$ (Buchoud et al., 2013) inducing a broadening and asymmetric Brillouin spectrum. In 


\section{Amsterdam '14}

this previous work, the assumption was made that the measured spectrum at $z_{k}$ is the combination of local stimulated Brillouin spectra $S_{e}(v)$ within $w$ centered on local Brillouin frequencies $v_{B}(x)$ :

$$
\tilde{G}\left(v, z_{k}\right) \cong \int_{z_{k}-w / 2}^{z_{k}+w / 2} S_{e}\left(v-v_{B}(x)\right) d x
$$

with $x$ a new spatial sampling. A first method proved the possibility of enhancement of the spatial sampling (Buchoud et al., 2013). However, this method missed some local strain variations that is crucial for the soil displacement quantification. Thus, we propose here method based on inverse method to improve the accuracy and the spatial sampling from the given matrix which is easily implemented.

\section{Parametric inverse methodology}

The model expressed in (2) can be rewritten in a classical parametric direct model as:

$$
G(v, z) \cong H\left(v_{B}(x)\right)+\epsilon
$$

with $H$ the direct operator described in (2) and $\epsilon$ the error linked to the model. $v_{B}(x)$ is the local Brillouin frequencies profile depending on the new spatial sampling $\Delta x$ and has to be estimated. The principal idea of this methodology is the minimization of the error defined by a cost function $C$ depending on the estimation of $v_{B}(x)$ :

$$
C\left(v_{B}(x)\right)=\left\|G(v, z)-H\left(v_{B}(x)\right)\right\|^{2}+\mu .\left\|\sum_{k=2}^{N-2} v_{B}\left(x_{k-1}\right)-v_{B}\left(x_{k+1}\right)\right\|^{2}
$$

The first term is the L2 norm between the data matrix and its estimation, while the second term is added to ensure the continuity of the local Brillouin frequencies $v_{B}(x)$. The parameter $\mu$ is the associated regularization term to be chosen. Note that if too much regularization is imposed, then the data and estimation would not fit and the first term would be too large. On the other hand, if $\mu$ is too small, the solution will be dominated by the contributions of data errors (Hansen et al. 2001). The parameter $N$ represents the number of local Brillouin frequencies to be estimated and is the ratio between the sensing lentgh and the chosen $\Delta x$. A trade-off between these two parameters can be controlled by the L-curve methodology. It is commonly used in geosciences (Chen et al., 1995) to optimize the regularization parameter $\mu$, known as the L-curve criterion. For several values of $\mu$, the L-curve represents the balance between the two terms of Eq. (1) in log scale. Then $\mu$ is found when the best trade-off between good data estimation and the a priori is guaranteed.

\section{Application on laboratory experiment}

A laboratory experiment has been carried out to study the distributed strain sensing chain in temperature controlled conditions (see details in Blairon et al., 2011). The configuration is presented in Figure 1.a. Two interrogators, Rayleigh scattering (Frogatt et al., 1998) and an industrial BOTDA devices are connected to one cable composed by several optical fibres. The cable is buried in a sand soil at two depths $h: 0.5 \mathrm{~m}$ and $1 \mathrm{~m}$ above a $1 \mathrm{~m}$ large metal plate. An underground cavity is generated using a hydraulic controlled actuator that moves the plate. For both interrogators, two state measurements are recorded: before (initial state $S_{0}$ ) and after (final state $S_{1}$ ) the actuator displacement. The Rayleigh scattering device provides a relative strain reference profile with a $3 \mathrm{~cm}$ spatial sampling. The profiles for the two depths are shown in Figure 1.b-c. in red curves. The displacement signature is composed by two traction zones (positive strain referenced at $-0.5 \mathrm{~m}$ and $0.7 \mathrm{~m}$ in distance) and a compressive zone (negative strain at $0.1 \mathrm{~m}$ ). For the depth $h=0.5 \mathrm{~m}$, over $1 \mathrm{~m}$ near the centre of the plate, the strain is going from $+200 \mu \mathrm{m} / \mathrm{m}$ to $-400 \mu \mathrm{m} / \mathrm{m}$, whereas at $h=1 \mathrm{~m}$, as it is far from the origin of the displacements, the strain gradient is less important from $+45 \mu \mathrm{m} / \mathrm{m}$ to $-270 \mu \mathrm{m} / \mathrm{m}$.

In the same time, Brillouin matrices were recorded by the BOTDA device with a spatial sampling $\Delta z=40 \mathrm{~cm}$ and integration base $w=1 \mathrm{~m}$. Figure $1 . \mathrm{d}$ shown in red stars and blue crosses the relative Brillouin shift $\Delta v_{B}(z)$ estimated by the BOTDA device for the two state, $\mathrm{S}_{0}$ and $\mathrm{S}_{1}$. A relative strain 


\section{Amsterdam '14}

profile can be estimated by using Eq. (1), the results for the two depths being plotted in Figure 1.b-c. in green curves. As these profiles have a resolution of $\Delta z=40 \mathrm{~cm}$, we linearly interpolate them in order to compare with the reference ones. As we can see, the displacement signature (positive and negative points) is retrieved. The extreme values are undervalued: for example, at $h=0.5 \mathrm{~m}$, instead having $400 \mu \mathrm{m} / \mathrm{m}$, the algorithm of the BOTDA device estimates $-250 \mu \mathrm{m} / \mathrm{m}$. In addition, due to $w=1 \mathrm{~m}$ and $\Delta z=40 \mathrm{~cm}$, the center of the plate and the minimum localization do not correspond. The Means Square Errors (MSE) between the strain reference and the strain profile estimated by the algorithm of the industrial BOTDA, shown in green in figure 1. e-f., highlight these limitations.

As our final goal is the displacements measurement into the structure, a precise strain is a crucial need. Thus, the proposed method is applied on the Brillouin matrices. The new spatial sampling $\Delta x$ is fixed at $5 \mathrm{~cm}$. The calculation time increases if $\Delta x$ is small and the sensing length is important. The first step is the estimation of the stimulated Brillouin spectrum $S_{e}(v)$, which can be found where strain is uniform within $w$. The regularization parameter is then optimized thanks the L-curve method in order to have the best ratio between measured/modelled data balance and continuity of frequencies. As the global frequencies profiles shown in Figures 1.b-c. are quite similar, $\mu=10$ is founded for both depths.

Relative Brillouin shifts with the resolution $\Delta x$ are then obtained and shown for the two state, $\mathrm{S}_{0}$ and $\mathrm{S}_{1}$ at $h=1 \mathrm{~m}$ in Figure 1.d. in continuous lines. The strain profiles estimated using Eq. (1) are shown in Figures 1.b-c. in blue curves. At $h=1 \mathrm{~m}$, our estimation and the strain reference are mixed up as well as at $h=0.5 \mathrm{~m}$, the negative part of the strain signature being well estimated. The minimum is better localized and its estimated value is closer however still undervalued. The MSE shown in blue in figure 1. e-f. decreases especially in high strain gradient areas. The proposed algorithm overcomes the difficult challenge to provide a precise strain profile with a centimetre spatial sampling from the measurement of Brillouin matrices with industrial 40-cm resolution BOTDA devices.

a.

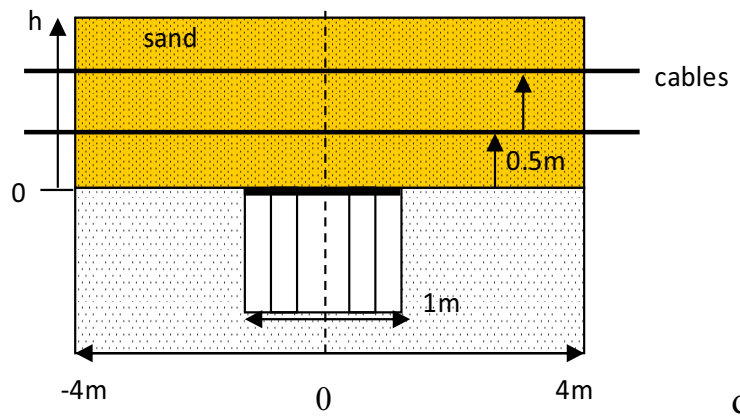

b.

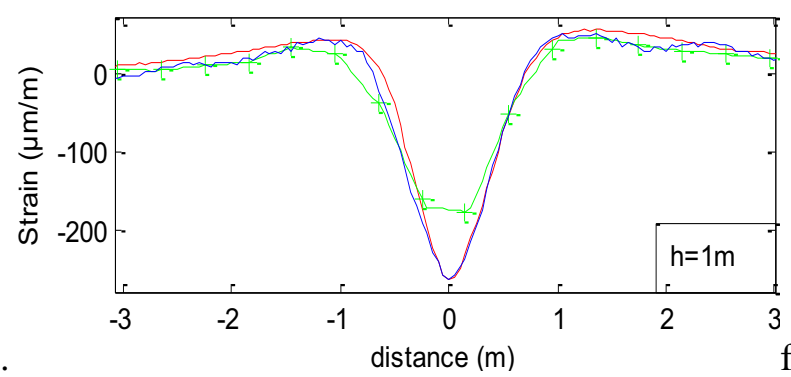

d.
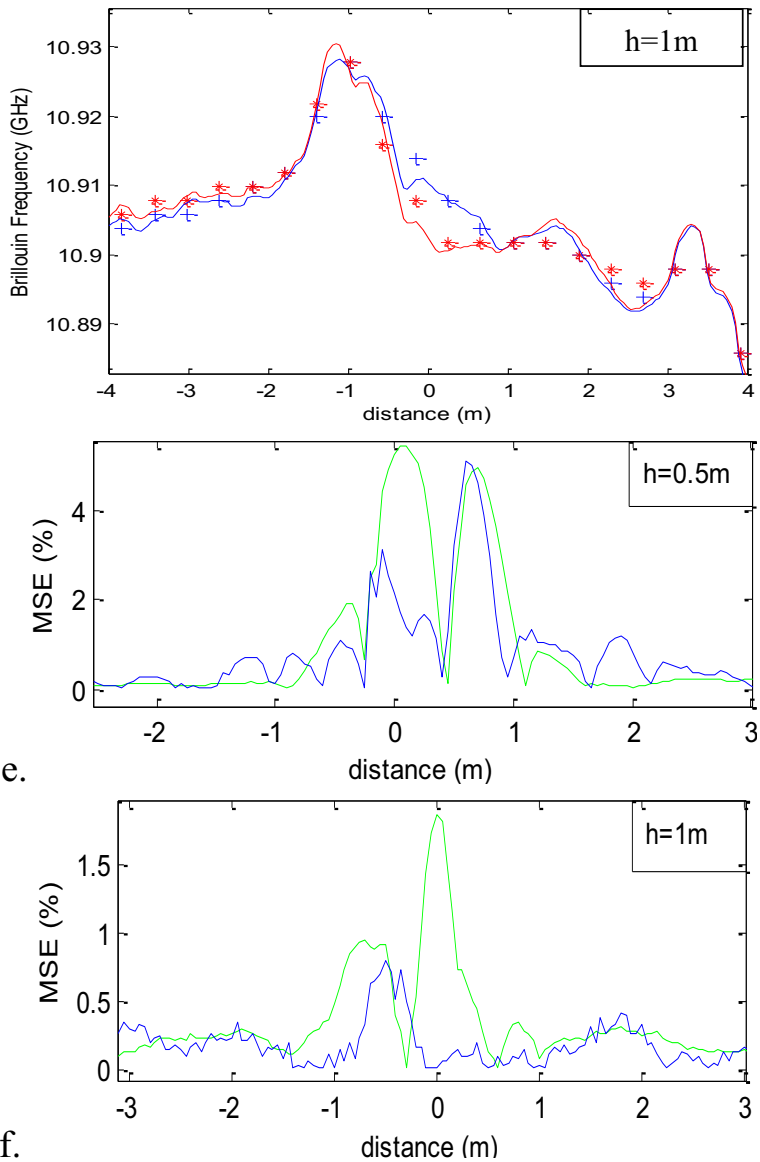

Figure 2. a). Scheme of the experiment: two cable at two depths $h=1 \mathrm{~m}$ and $0.5 \mathrm{~m}$ from the metal plate. $\mathrm{b}$ and c) Strain profiles provided by the Rayleigh device (red - refernce); the BOTDA algorithm (green - interpolated from $40 \mathrm{~cm}$ spatial rezolution) and the inverse method (blue - with a new spatial 


\section{Amsterdam '14}

rezolution $\Delta x=5 \mathrm{~cm}$ ) for the two depths $h=0.5 \mathrm{~m}(\mathrm{~b})$ and $h=1 \mathrm{~m}(\mathrm{c}) . \mathrm{d})$. Estimation of the Brillouin frequencies at $h=1 \mathrm{~m}$ : provided by the BOTDA $(+)$ state $\mathrm{S}_{0} ;\left(^{*}\right)$ state $\mathrm{S}_{1}$; and the inverse method : (-) $\mathrm{S}_{0}$; (-) $\mathrm{S}_{1}$. e and f) MSE between the strain reference profile (Rayleigh device) and the strain profile estimated by the algorithm of the industrial BOTDA (green) respectively the strain profile estimated by our inverse method (blue) for the two depths $h=0.5 \mathrm{~m}$ (e) and $h=1 \mathrm{~m}$ (f).

\section{Conclusion}

The classical BOTDA provides a strain measurement profile with a $40 \mathrm{~cm}$ spatial sampling. Based on previous works, the interpretation of the distortion of the Brillouin spectrum relies in a direct model to retrieve local Brillouin frequencies into the measured Brillouin spectra. A parametric inverse methodology is proposed to estimate those local Brillouin frequencies depending on distance with a new spatial sampling chosen by the user. The best trade-off between data reconstruction and the $a$ priori on the continuity of the Brillouin profile is found thanks the L-curve methodology. On controlled conditions, our method has been tested on real data acquired by an industrial BOTDA device. From a $40 \mathrm{~cm}$ spatial resolution recorded data, the strain profile is estimated with lower spatial resolution chosen here to be $5 \mathrm{~cm}$. In term of accuracy, our strain estimation is similar to the strain reference profile provided by a Rayleigh scattering device. Whereas the algorithm of industrial BOTDA devices does not take into account the hidden information into the Brillouin spectra, we enlighten its lack of accuracy. The challenge of combining centimetre spatial sampling over kilometers with high strain accuracy has thus been successfully performed thanks to a parametric inverse methodology. Future work consists to estimate the displacements into the structure from the strain measurements.

\section{References}

Bao X., Dhliwayo J., Heron N., Webb D.J. and D.A. Jackson. 1995. Experimental and theorical studies on a distributed temperature sensor based on Brillouin scattering, Journal of Lightwave Technology, Vol. 13, No. 7, pp.1340-1348.

Blairon S., Hénault J-M., Buchoud E., Vinceslas G., Delepine-Lesoille S., 2011. Truly distributed optical fiber extensometers for geotechnical structure monitoring (dikes and underground repository): Influence of sensor external coating, $8^{\text {th }}$ international symposium of Field Measurement in Geomecanics, Expanded Abstract, Berlin, Germany, September.

Buchoud E., S. Blairon , G. D’Urso, J.-M. Hénault, A. Girard, Vrabie V.and J. I. Mars , 2012. Detection of ground movement using the shape of Brillouin spectrum, Near Surface Geoscience, expended abstract, Paris, France, September.

Buchoud E., Vrabie V., J. I. Mars, G. D’Urso, A. Girard, S. Blairon, and J.-M. Hénault, 2013. Enhancement of an optical fiber sensor: source separation based on Brillouin spectrum. IEEE Access.

Chen L. Y., J. T. Chen, H.-K. Hong, and C. H.Chen, 1995. Application of Cesaro mean and the Lcurve for the deconvolution problem. Soil Dynamics and Earthquake Engineering, Vol. 14, pp. 361373.

Frogatt M. and Moore J., 1998. High-spatial-resolution distributed strain measurement in optical fiber with Rayleigh scatter. Applied Optics, Vol.37, No.10, pp. 1735-1740.

Hansen PC, 2001. The L-curve and its use in the numerical treatment of inverse problems. In: Johnston P, editor. Southampton: WIT Press.

Lanticq V., Gabet G., Taillade F., Delepine-Lesoille S., 2009, Distributed Optical fiber sensors for structural health monitoring: upcoming challenges, chapter 9, in "Optical Fiber, New Developments".

Nikles M., Thevenaz L., Robert P.A, 1997. Brillouin gain spectrum characterization in single-mode optical fibers. Journal of Ligthwave technology, Vol.15, No.10, pp. 1842-1851. 